\title{
Finite element nonlinear transient modelling of carbon nanotubes reinforced fiber/polymer composite spherical shells with a cutout
}

https://doi.org/10.1515/ntrev-2019-0039

Received Mar 18, 2019; accepted Aug 22, 2019

\begin{abstract}
This study dealt with geometrically nonlinear transient behaviors of carbon nanotube/fiber/polymer composite (CNTFPC) spherical shells containing a central cutout. A multiscale analysis using the Hewitt and Malherbe model was performed to determine the carbon nanotube (CNT) weight ratios, thickness-radius ratios, thickness-length ratios of CNTs, and cutout sizes. Based on the first-order shear deformation plate theory (FSDT), the Newmark method and Newton-Raphson iteration were used for the nonlinear dynamic solution. The proposed approach in this study has been verified by previous studies. Parametric results showed the significance of a proper CNT ratio and curvature for better structural performance on the nonlinear dynamic behaviors of CNTFPC spherical shells with a cutout.
\end{abstract}

Keywords: CNTFPC spherical shell; Finite element nonlinear transient modelling; carbon nanotube; multiscale model; CNT weight ratio

\section{Introduction}

Carbon-based nanomaterials have been applied to various engineering fields because of their excellent mechanical properties. In some of these fields, compound and application technologies of carbon nanotubes (CNTs) have greatly increased the need for multifunctional materials for semiconductors, composites, flexible displays, etc. The reason for this is that CNTs have higher Young's modulus and ten-

\footnotetext{
${ }^{\star}$ Corresponding Author: Sang-Youl Lee: Department of Civil Engineering, Andong National University, 1375 Gyeongdong-ro, Andong, Kyoungsangbuk-do 36729, Korea; Email: lsy@anu.ac.kr;

Tel: +82 54 820-5847; Fax: +82 54 820-6625

Ji-Gwang Hwang: Department of Civil Engineering, Andong National University, 1375 Gyeongdong-ro, Andong, Kyoungsangbuk-do 36729, Korea
}

๖ Open Access. ๑ 2019 autor, published by De Gruyter.

(cc) BY sile strength than those of existing materials. For example, CNTs are a hundred times stronger than steel, but are three to five times lighter. Moreover, the strain at the breaking point of CNTs is about $10 \%$, which is much higher than that of composite materials. For these reasons, theoretical and experimental studies related to CNTs have been performed by a number of investigators. However, most of these studies were focused on dispersion or compound technologies from a micromechanical point of view [1-5].

The macromechanical performances of CNTs have been studied by several researchers for carbon nanotube reinforced composite (CNTRC) beams or plates. Wattanasakulpong and Ungbhakorn [6] dealt with the closed forms for the bending, buckling, and vibration responses of CNTRC beams resting on an elastic foundation. Zhu et al. [7] studied the static and free vibration of functional graded(FG) CNTRC plates according to the first-order shear deformation theory (FSDT), and the study was extended to the Levy method for the natural frequency analysis of FG-CNT composites under inplane loads using the higher-order shear deformation plate theory (HSDT) [8]. The multi-scale mechanical behaviors of three-phase CNTs/fiber/polymer laminated composites have also been studied by a few investigators [9]. In the studies, the effective material properties of the CNT/fiber/polymer composite (CNTFPC) structures were computed using a combination of the Halpin-Tsai scheme and a micromechanical approach. However, these studies dealt with CNTRC or CNTFPC rectangular or skew-type structures, which are not considered in geometrical curvature effects.

Recently, structural analyses of cylindrical panels or shells made of CNTRCs or CNTFPCs have been carried out for various parameters. For example, GarcíaMacías et al. [10] performed a buckling analysis of FG-CNTreinforced cylindrical panels subjected to axial compression and shear. Mirzaeia and Kianib [11] studied the free vibration of FG-CNT-reinforced composite cylindrical panels. Zhang et al. [12] investigated the static and dynamic behaviors of FG-CNT-reinforced cylindrical panels. These 
studies were further extended to deal with the dynamic instability problems of FG-CNTRC cylindrical panels [13-15]. However, all of these studies were limited in their coverage of the CNT effects of CNTRC cylindrical panels without a cutout. Cutout in composite structures has significant effects on the dynamic behaviors, especially for the geometrical non-linearity. To the best of this author's knowledge, a nonlinear transient analysis of CNTFPC laminated spherical shells with a cutout is not yet widely available in the literature. Bhardwaj et al. [16] dealt with the nonlinear static and dynamic analysis of CNT reinforced composite plate-type structures without a cutout. The nonlinear static or dynamic behaviors of laminated composites with a cutout has been studied by other investigators; however, most relevant studies have dealt with the typical composites, which are not reinforced by CNTs [17-19].

In this study, the multi-scale nonlinear dynamic behaviors of CNTFPC laminated spherical shells with a central cutout was investigated. The multiscale analysis using the modified Halpin-Tsai model were performed on the basis of the FSDT. Parametric examples were focused on the correlation between radius-length ratios, cutout sizes, and CNT weight ratios. The interactions between the parameters also showed different trends for the cutout sizes, which are difficult to predict. The significance of each parameter in predicting the geometrically dynamic nonlinearity of CNTFPC spherical shells with a cutout is investigated.

\section{Theoretical formulation}

\subsection{Multiscale formulation for CNTFPC shells}

To perform a multiscale analysis of CNT/fiber/polymer multi-phase composites (referred to as CNTFPC), we used the modified Halpin-Tsai model (refferred as Hewitt and Malherbe model) and micromechanical approaches. CNTFPC shells were dealt with in this study as they were assumed to have a perfect distribution of CNTs and to be impregnated with polymer. First, the effective elastic modulus of CNT-reinforced resins (referred to as CNTRC) can be determined using the Halpin-Tsai equation as [20]

$$
\begin{aligned}
E^{c n r} & =E^{r e}\left[\frac{3}{8}\left(\frac{1+2\left(l^{c n t} / d^{c n t}\right) \gamma_{d l} V_{c n t}}{1-\gamma_{d l} V_{c n t}}\right)\right. \\
& \left.+\frac{5}{8}\left(\frac{1+2 \gamma_{d d} V_{c n t}}{1-\gamma_{d d} V_{c n t}}\right)\right],
\end{aligned}
$$

where,

$$
\gamma_{d d}=\frac{\left(E_{11}^{c n t} / E^{r e}\right)-\left(d^{c n t} / 4 t^{c n t}\right)}{\left(E_{11}^{c n t} / E^{r e}\right)+\left(d^{c n t} / 2 t^{c n t}\right)},
$$

$$
\gamma_{d l}=\frac{\left(E_{11}^{c n t} / E^{r e}\right)-\left(d^{c n t} / 4 t^{c n t}\right)}{\left(E_{11}^{c n t} / E^{r e}\right)+\left(l^{c n t} / 2 t^{c n t}\right)},
$$

where, $E^{c n r}$ is the effective elastic modulus of CNTreinforced resins, and $E^{r e}$ and $E_{11}^{c n t}$ are Young's modulus of resin and single-walled carbon nanotubes (SWCNTs) or multi-walled carbon nanotubes (MWCNTs), respectively. $d^{c n t}, l^{c n t}$, and $t^{c n t}$ are the diameter, length, and thickness of SWCNTs or MWCNTs. The Possion's ratio $\left(v_{12}^{c n r}\right)$ and mass density $\left(\rho^{c n r}\right)$ are determined from the rule of mixture as

$$
\begin{aligned}
& v_{12}^{c n r}=v^{c n t} V_{c n t}+v^{r e} V_{r e} \\
& \rho^{c n r}=\rho^{c n t} V_{c n t}+\rho^{r e} V_{r e}
\end{aligned}
$$

where, $v^{\text {cnt }}$ and $v^{r e}$ are Possion's ratios of CNTs and resin, and $\rho^{c n t}$ and $\rho^{r e}$ are mass densities of CNTs and resin, and $V_{r e}$ is the volume fraction of resin, respectively. In Eqs. (9)(10), the volume fraction $\left(V_{c n t}\right)$ of CNTs can be determined as

$$
V_{c n t}=\frac{w^{c n t}}{w^{c n t}+\left(\rho^{c n t} / \rho^{r e}\right)-\left(\rho^{c n t} / \rho^{r e}\right) w^{c n t}},
$$

where, $w^{\text {cnt }}$ denotes the weight ratio of the SWCNTs or MWCNTs.

Next, the CNT-reinforced resins are reinforced again with fibers, which are the CNTFPC. The effective longitudinal Young's modulus $\left(E_{11}\right)$ and the Poisson's ratio $\left(v_{12}\right)$ of the CNTFPC spherical shells are written using the rule of mixture as

$$
\begin{aligned}
& E_{11}=E^{f} V^{f}+E^{c n r}\left(1-V^{f}\right), \\
& v_{12}=v_{12}^{f} V^{f}+v_{12}^{c n r}\left(1-V^{f}\right) .
\end{aligned}
$$

where, $E^{f}, v_{12}^{f}$ and $V^{f}$ mean the Young's modulus, Poisson's ratio and the volume fraction of the fiber, respectively. The transverse Young's modulus $\left(E_{22}\right)$ is given by

$$
E_{22}=E^{r e}\left(\frac{1+\chi_{1} \eta_{1} V^{f}}{1-\eta_{1} V^{f}}\right), \eta_{1}=\frac{\left(E^{f} / E^{c n r}\right)-1}{\left(E^{f} / E^{c n r}\right)+\chi_{1}} .
$$

The in-plane shear modulus $\left(G_{12}\right)$ determined from Halphin-Tsai formulation is the similar form as that obtained using Eq. (8).

$$
G_{12}=G^{r e}\left(\frac{1+\chi_{2} \eta_{2} V^{f}}{1-\eta_{2} V^{f}}\right), \eta_{2}=\frac{\left(G^{f} / G^{c n r}\right)-1}{\left(G^{f} / G^{c n r}\right)+\chi_{2}},
$$

where, $G^{r e}$ and $G^{c n r}$ are the shear modulus of the resin and CNT-reinforced resins, and $G^{f}$ is the shear modulus of the fiber. Hewitt and Malherbe proposed a modified equation for the calibration of $\chi_{2}$ and $\chi_{2}$ for a high volume fraction,

$$
\chi_{1}=2.0+40\left(V^{f}\right)^{10}, \chi_{2}=1.0+40\left(V^{f}\right)^{10}
$$

It should be noted that the results obtained from Eq. (9) were in good agreement with the experimental data from Hashin (1970) [21]. For this reason, this study applied the modified Halpin-Tsai equation from Hewitt and Malherbe for the effective in-plane shear modulus. 


\subsection{Nonlinear transient modelling for CNTFPC spherical shells}

In this study, we developed a finite element nonlinear dynamic model based on the FSDT. For the sake of completeness, nonlinear transient formulations are presented here. Figure 1 shows the geometry and coordinate system $\left(\xi_{1}, \xi\right.$, $\zeta$ ) of the laminated CNTFPC spherical shell containing a central cutout. Based on the shell theory of Sanders [22], the strain components for the spherical shells with the principal radii of curvature $R_{1}$ and $R_{2}$ can be written as [23]

$$
\begin{aligned}
& \left\{\begin{array}{c}
\epsilon_{\xi_{1} \xi_{1}} \\
\epsilon_{\xi_{2} \xi_{2}} \\
\gamma_{\xi_{2} \xi_{3}} \\
\gamma_{\xi_{1} \xi_{3}} \\
\gamma_{\xi_{1} \xi_{2}}
\end{array}\right\} \\
& =\left\{\begin{array}{c}
u_{0, \xi_{1}}+w_{0, R_{1}}+\frac{1}{2}\left(w_{0, \xi_{1}}\right)^{2}+\zeta \phi_{\xi_{1}, \xi_{1}} \\
v_{0, \xi_{2}}+w_{0, R_{2}}+\frac{1}{2}\left(w_{0, \xi_{2}}\right)^{2}+\zeta \phi_{\xi_{2}, \xi_{2}} \\
w_{0, \xi_{2}}+\phi_{\xi_{2}} \\
w_{0, \xi_{1}}+\phi_{\xi_{1}} \\
u_{0, \xi_{2}}+v_{0, \xi_{1}}+w_{0, \xi_{1}} w_{0, \xi_{2}}+\zeta \phi_{\xi_{1}, \xi_{2}} \phi_{\xi_{2}, \xi_{1}}
\end{array}\right\}
\end{aligned}
$$

where $\left(u_{0}, v_{0}, w_{0}\right)$ denote the displacements of a point $\left(\xi_{1}\right.$, $\xi, 0)$ on the mid-surface of the shell, and $\phi_{\xi_{1}}$ and $\phi_{\xi_{2}}$ denote the rotations of a normal to the reference surface, respectively. From the nonlinear strain-displacement relationship, the stress resultants and the equations of motion of the CNTFPC spherical shell. The additional details consult the reference by Sanders [22].

The nonlinear equation of motion at time $p+1$ can be reduced to the fully discretized from using Newmark's scheme:

$$
\left[\tilde{K}\left(\{\delta\}_{p+1}\right)\right]\{\delta\}_{p+1}=\{\tilde{F}\}_{p, p+1},
$$

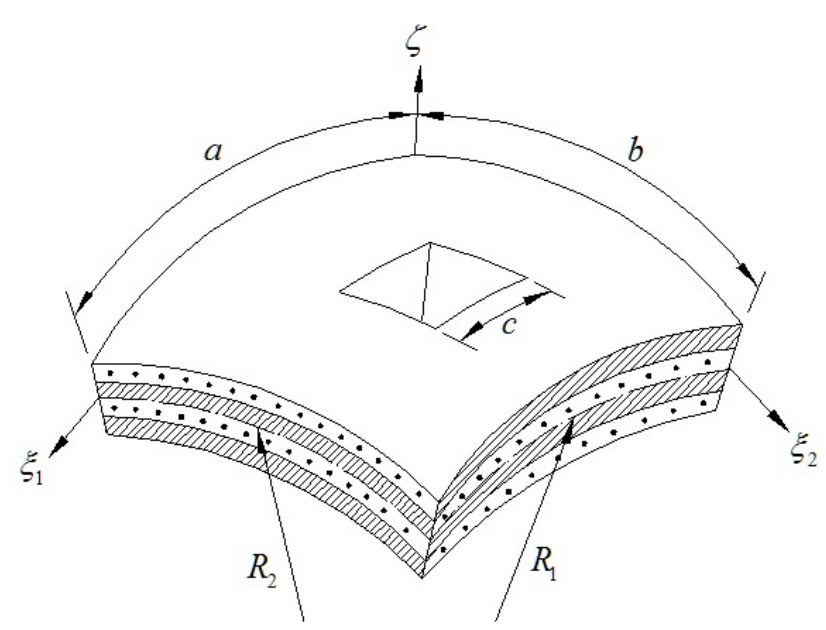

Figure 1: A laminated CNTFPC spherical shell with a central cutout where,

$$
\begin{aligned}
& {\left[\tilde{K}\left(\{\delta\}_{p+1}\right)\right]=\left[K\left(\{\delta\}_{p+1}\right)\right]+\kappa_{1}\left[M\left(\{\delta\}_{p+1}\right)\right]} \\
& \{\tilde{F}\}_{p, p+1}=\{F\}_{p+1}+[M]_{p+1}\left(\kappa_{1}\{\delta\}_{s}+\kappa_{2}\{\dot{\delta}\}_{s}\right. \\
& \left.+\kappa_{3}\{\ddot{\delta}\}_{s}\right)
\end{aligned}
$$

where, $\left[K\left(\{\delta\}_{p+1}\right)\right],\left[M\left(\{\delta\}_{p+1}\right)\right]$, and $\{F\}_{p+1}$ denote the element stiffness matrix, the element mass matrix, and the external force vector at time $t_{p+1}$, respectively. The parameters $\kappa_{1}-\kappa_{3}$ are used to compute the effective stiffness matrix $\left[\tilde{K}\left(\{\delta\}_{p+1}\right)\right]$ and the effective force vector $\{\tilde{F}\}_{p, p+1}$. At the end of each time step, the new velocity vector $\{\dot{\delta}\}_{s+1}$ and acceleration vector $\{\ddot{\delta}\}_{s+1}$ are determined using the Newmark's scheme [24]. Using the Newton-Raphon iterative method, the solution at the sth iteration, $\{\delta\}_{p+1}^{s}$ can be expressed as

$$
\{\hat{R}\}\left(\{\delta\}_{p+1}\right) \equiv\left[\tilde{K}\left(\{\delta\}_{p+1}\right)\right]\{\delta\}_{p+1}-\{\tilde{F}\}_{p, p+1}=0,
$$

where, $\{\hat{R}\}$ is a nonlinear function of $\{\delta\}_{p+1}$. Expanding $\{\hat{R}\}$ in Taylor's series about $\{\delta\}_{p+1}^{s}$, the following iterative equation is obtained:

$$
\begin{gathered}
0=\{\hat{R}\}_{p+1}^{s}+\left(\left[\tilde{K}^{T}\left(\{\delta\}_{p+1}^{s}\right)\right]\right)\{\bar{\delta}\}+O\left(\{\bar{\delta}\}^{2}\right), \\
{\left[\tilde{K}^{T}\left(\{\delta\}_{p+1}^{s}\right)\right] \equiv\left[\frac{\partial\{R\}}{\partial\{\delta\}}\right]_{p+1}^{s},} \\
\{\hat{R}\}_{p+1}^{s}=\left[\tilde{K}\left(\{\delta\}_{p+1}^{s}\right)\right]\{\delta\}_{p+1}^{s}-\{\tilde{F}\}_{p, p+1}
\end{gathered}
$$

where, $O(\cdot)$ and $\left[\tilde{K}^{T}\right]$ are the higher-order terms and the tangent stiffness matrix. The assembled equations are solved for the incremental displacement vector after imposing the boundary and initial conditions.

\section{Parametric results and discussion}

\subsection{Verification}

Table 1 shows the detailed properties of the CNTs, resins, and fibers used for parametric examples. Figure 2 shows the comparisons of non-dimensionalized nonlinear transient displacement of composite spherical shells under uniformly distributed load. It can be observed from the figure that the present results agreed well with those reported by other investigators. Figure 2 shows nondimensionalized nonlinear transient displacements of SWCNTs reinforced composite plates for different lengthdiameter ratios and weight ratios of SWCNTs. It is evident 
Table 1: Material and geometrical properties of the materials used in this study

\begin{tabular}{|c|c|c|c|c|}
\hline Material & Source & Symbol & Value & Definition \\
\hline Material I & [18] & $\begin{array}{c}E_{11} \\
E_{22} \\
G_{12} \\
\rho \\
v_{12}\end{array}$ & $\begin{array}{c}25 E_{22} \\
10^{6} \mathrm{~N} / \mathrm{cm}^{2} \\
0.5 E_{22} \\
1 \mathrm{Ns}^{2} / \mathrm{cm}^{4} \\
0.25\end{array}$ & $\begin{array}{l}\text { Longitudinal Young's modulus } \\
\text { Transverse Young's modulus } \\
\text { In-plane shear modulus } \\
\text { Mass density } \\
\text { Possion's ratio }\end{array}$ \\
\hline Epoxy resin & [25] & $\begin{array}{l}E^{e p} \\
\rho^{p m} \\
\nu^{p m}\end{array}$ & $\begin{array}{c}2.72 \mathrm{GPa} \\
1,200 \mathrm{~kg} / \mathrm{m}^{3} \\
0.33\end{array}$ & $\begin{array}{l}\text { Young's modulus of epoxy resin } \\
\text { Mass density of epoxy resin } \\
\text { Possion's ratio of epoxy resin }\end{array}$ \\
\hline SWCNT & [3] & $\begin{array}{l}E_{11}^{c n t} \\
\rho^{c n t} \\
v^{c n t} \\
t^{c n t} \\
d^{c n t} \\
l^{c n t}\end{array}$ & $\begin{array}{c}640 \mathrm{GPa} \\
1,350 \mathrm{~kg} / \mathrm{m}^{3} \\
0.33 \\
0.34 \mathrm{~nm} \\
1.4 \mathrm{~nm} \\
25 \mu \mathrm{m}\end{array}$ & $\begin{array}{c}\text { Young's modulus of SWCNT } \\
\text { Mass density of SWCNT } \\
\text { Possion's ratio of SWCNT } \\
\text { Thickness of SWCNT } \\
\text { Diameter of SWCNT } \\
\text { Length of SWCNT }\end{array}$ \\
\hline MWCNT & [3] & $\begin{array}{l}E_{11}^{c n t} \\
\rho^{c n t} \\
v^{c n t} \\
t^{c n t} \\
d^{c n t} \\
l^{c n t}\end{array}$ & $\begin{array}{c}400.0 \mathrm{GPa} \\
1,350 \mathrm{~kg} / \mathrm{m}^{3} \\
0.33 \\
0.34 \mathrm{~nm} \\
20.0 \mathrm{~nm} \\
50 \mu \mathrm{m}\end{array}$ & $\begin{array}{l}\text { Young's modulus of MWCNT } \\
\text { Mass density of MWCNT } \\
\text { Possion's ratio of MWCNT } \\
\text { Thickness of MWCNT } \\
\text { Diameter of MWCNT } \\
\text { Length of MWCNT }\end{array}$ \\
\hline E-Glass fiber & [25] & $\begin{array}{l}E^{f} \\
\rho^{f} \\
v^{f}\end{array}$ & $\begin{array}{c}69.0 \mathrm{GPa} \\
1,200 \mathrm{~kg} / \mathrm{m}^{3} \\
0.2\end{array}$ & $\begin{array}{l}\text { Young's modulus of E-Glass fiber } \\
\text { Mass density of E-Glass fiber } \\
\text { Possion's ratio of E-Glass fiber }\end{array}$ \\
\hline
\end{tabular}

that the induced displacements agreed well with those reported by Bhardwaj et al. [16].

Figure 4 shows the comparisons of nonlinear transient displacements of SWCNT reinforced composite spherical shells $(R / a=10)$ for different multiscale models. The CNTFPC spherical shells with all simply supported edges were laminated as $[0 / 90]_{s}$, and the results determined from different multi-scale formulations were compared. It may be noted that only the Halpin-Tsai model was corrected for a volume fraction of less than 0.5 . For this reason, we used the multi-scale model modified from Hewitt and Malherbe equation as presented in Eqs.(8)-(9) for $E_{22}$ and $G_{12}$. For $V^{f}=0.8$, the maximum displacement using the conventional Halpin-Tsai equation was about $22 \%$ higher than those obtained using the modified equation by Hewitt and Malherbe. Therefore, for greater accuracy, all the subsequent induced results were based on the modified Halpin-Tsai equation.

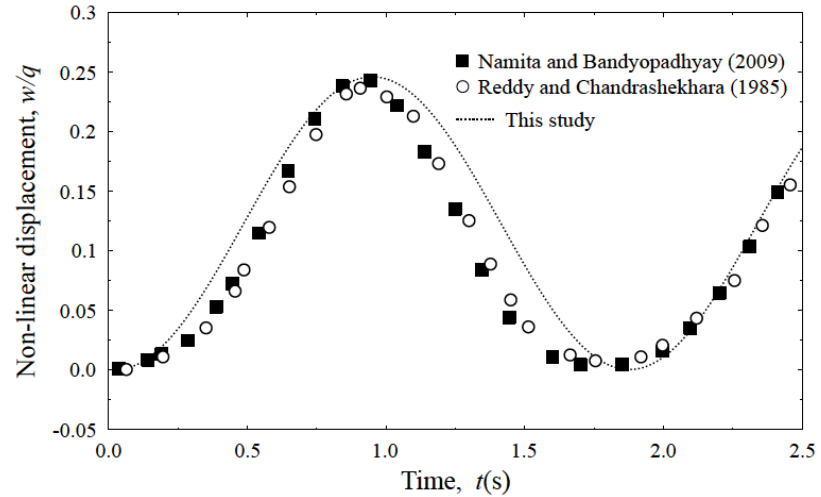

Figure 2: Non-dimensionalized nonlinear transient displacement $(w / q)$ of composite spherical shells under uniformly distributed load ([0/90], Material I) 


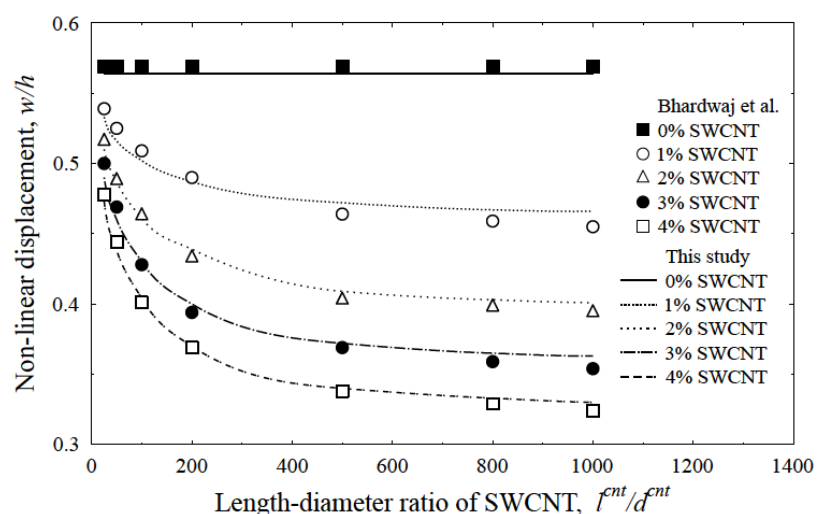

Figure 3: Non-dimensionalized nonlinear transient displacement $(w / h)$ of SWCNT reinforced composite plates for different lengthdiameter ratios of SWCNT ([0/90/90/0])

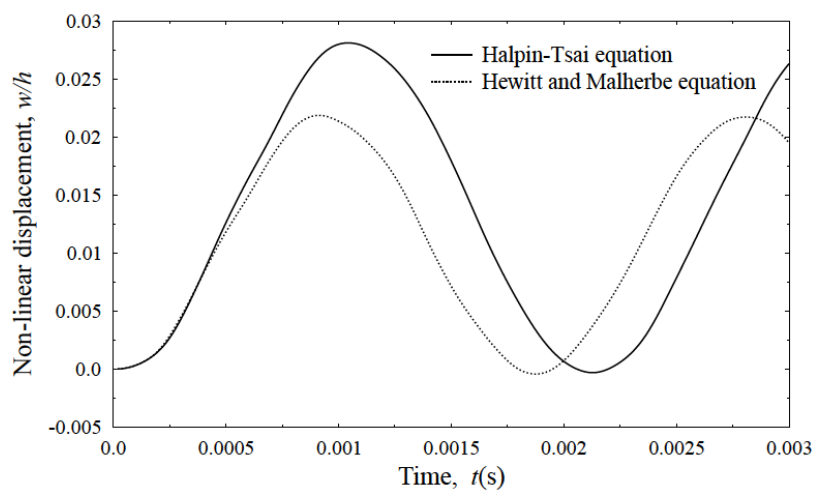

Figure 4: Comparison of nonlinear transient displacements $(w / h)$ of SWCNT reinforced composite spherical shells $(R / a=10)$ for different multiscale models ( $q=1.0 \mathrm{MPa},[0 / 90]_{s}, V^{f}=0.8$, $w^{c n t}=1.0 \%$ no cutout .

\subsection{CNTFPC spherical shells without a cutout}

Figure 5 represents nonlinear transient displacements of $[0 / 90]_{s}$ SWCNT reinforced composite flat plates and spherical shells for different the SWCNT weight ratios. All parameters were compared for shells without a cutout. The maximum displacements occurred at earlier time and dereased as the SWCNT ratios increased. For both plates and shells, the trends were similar to each other. This is clearly due to increased stiffness effects as the SWCNT weight ratio increases. The SWCNT reinforcement plays a role in increased stiffness and lead to the higher frequency of plates and shells. Nonlinear transient displacement for shells are lower than those of plates because of membrane force effects resulted from the curvature of the shell. It can be also be seen from the figure that small SWCNT weight ratios of $1-2 \%$ resulted in the noticeable reduction of nonlinear dynamic displacements. On the other hand, the differences in induced displacements for increased MWCNT ra-

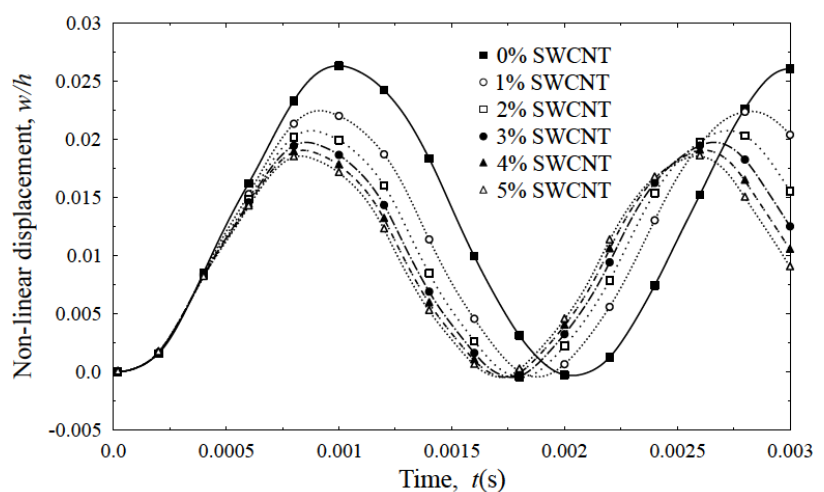

(a) Flat plates

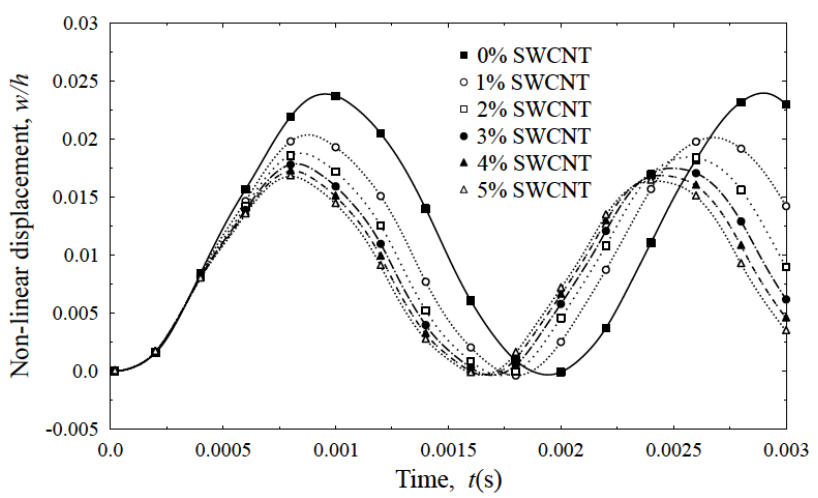

(b) Spherical shells $(\mathrm{R} / \mathrm{a}=5.0)$

Figure 5: Nonlinear transient displacements $(w / h)$ of SWCNT reinforced composite flat plates and spherical shells ( $q=1.0 \mathrm{MPa}$, $[0 / 90]_{s}, V^{f}=0.8$, no cutout)

tios are negligible for flat plates as shown in Figure 6. For the shell with $R / a=5.0$, the induced displacement for the MWCNT weight ratio of $1.0 \%$ is extremely lower than that for the shell without MWCNT reinforcement. For the MWCNT weight ratio of more than $1.0 \%$, the induced displacements are close to each other as shown in Figure 6(b). It can be concluded from the results that small MWCNT ratios result in better rigidity against dynamic loading, especially for the spherical shell.

Figure 7 shows induced nonlinear transient displacements of $[0 / 90]_{s}$ CNTFPC spherical shells with 1.0\% SWCNT reinforcement for different radius-length ratios. The maximum displacement occurred at earlier time as the radius-length ratios decreased. As mentioned earlier, this is due to curvature effects such as the occurrence of membrane forces as the radius-length decreases. In this case, the membrane force effects also play a role in increased stiffness and lead to the higher frequency of spherical shells. 


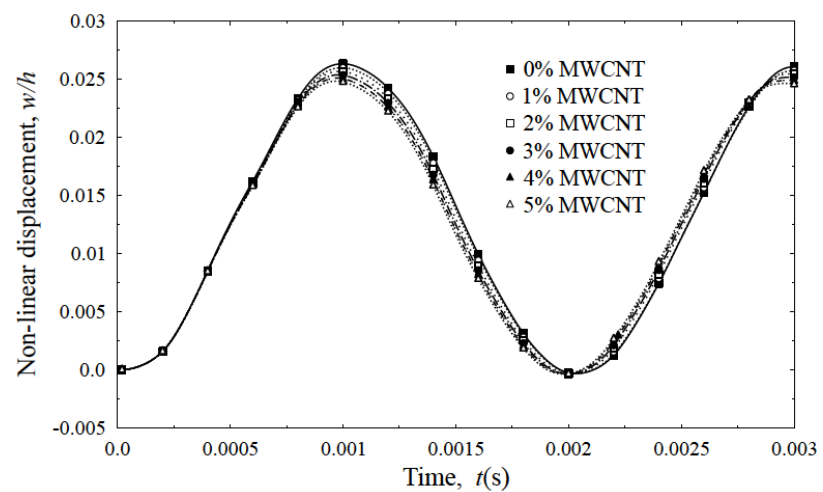

(a) Flat plates

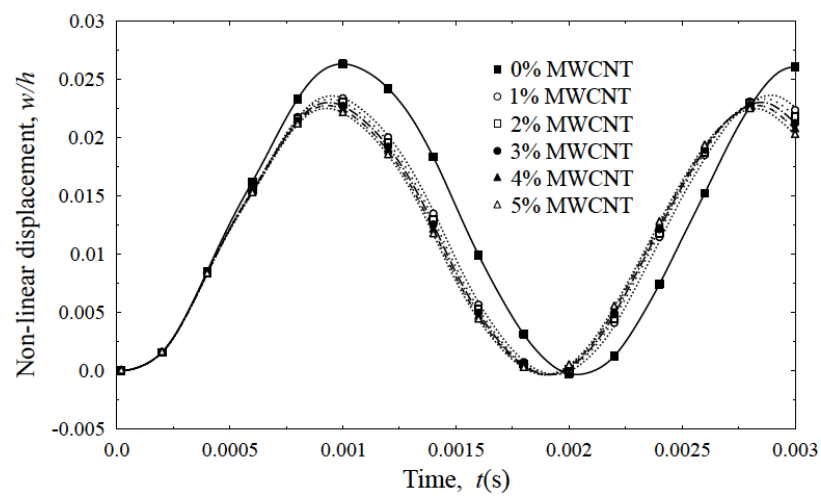

(b) Spherical shells $(\mathrm{R} / \mathrm{a}=5.0)$

Figure 6: Nonlinear transient displacements $(w / h)$ of MWCNT reinforced composite flat plates and spherical shells $(q=1.0 \mathrm{MPa}$, $[0 / 90]_{s}, V^{f}=0.8$, no cutout)

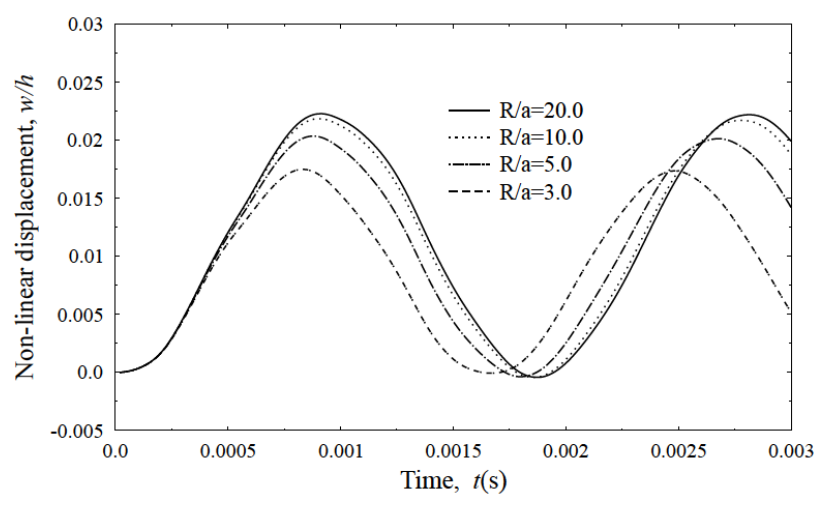

Figure 7: Nonlinear transient displacements $(w / h)$ of SWCNT reinforced composite spherical shells for different radius-length ratios $\left(q=1.0 \mathrm{MPa},[0 / 90]_{s}, V^{f}=0.8, w^{c n t}=1.0 \%\right.$, no cutout $)$

\subsection{CNTFPC spherical shells with a cutout}

Figure 8 represents nonlinear dynamic behaviors of $[45 /-45]_{s}$ SWCNT reinforced composite spherical shells for different cutout sizes. A distributed step loading of loading of magnitude $q=1.0 \mathrm{MPa}$ is applied at time $t=0$.

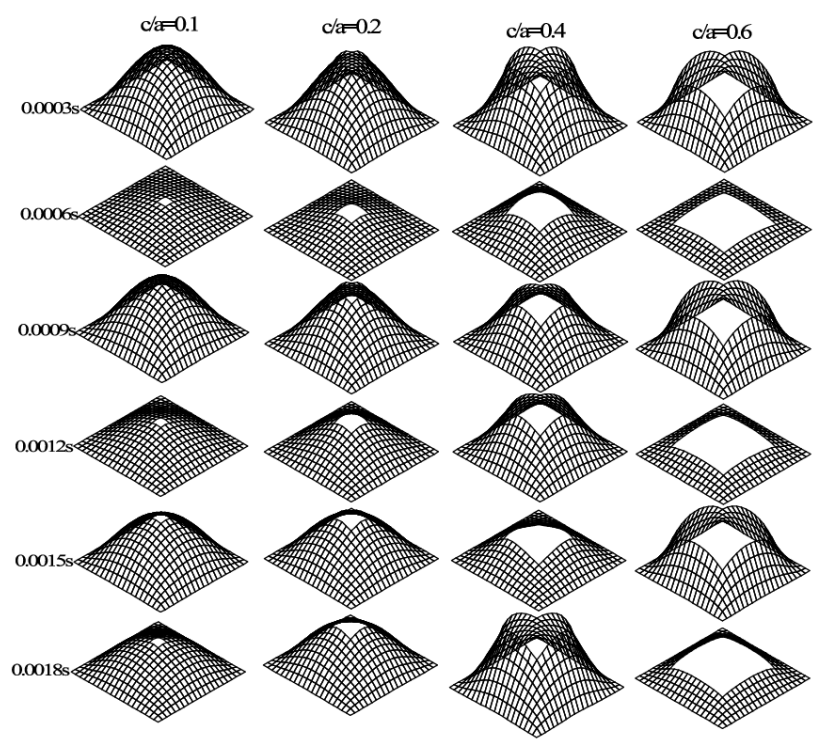

Figure 8: Nonlinear dynamic behaviors $(w / h)$ of SWCNT reinforced composite spherical shells for different cutout sizes ( $q=1.0 \mathrm{MPa}$, $\left.[45 /-45]_{s}, V^{f}=0.8, w^{c n t}=1.0 \%\right)$

The deformed shape of CNTFPC spherical shells for the different cutout sizes is a dominant factor in identifying dynamic characteristics of SWCNT reinforced laminates under a suddenly applied distributed load. It can be observed from the figure that the deformed shapes at different time steps are significantly different for the cutout size of more than $c / a=0.6$. The amplitude of overall displacements for bigger cutout size is larger than that of small cutout size due to the mass loss.

Figures 9 shows the interactions of maximum nonlinear displacements between the cutout sizes, SWCNT weight ratios, and radius-length ratios of CNTFPC spherical shells. For increased SWCNT weight ratios, the maximum nonlinear transient displacements sharply decreased as radius-length ratios $(R / a)$ decreased, especially for $R / a<5.0$. The SWCNT reinforcements showed greater enhancement to the nonlinear dynamic flexural rigidity, especially for shells with large curvatures. On the other hand, effects on cutout areas were relatively small for the flat panels. For spherical shells with $R / a=5.0$ and $c / a=$ 0.4 cutout area, the induced nonlinear displacements were similar to that of shells with $c / a=0.4$ cutout area. On the other hand, the nonlinear transient displacements of shells with $R / a>5.0$ asymptotically approached a fixed value for increased the SWCNT weight ratios $(>2.0 \%)$. These results lead us to conclude that spherical shells with $R / a>5.0$ can be considered as having similar nonlinear dynamic rigidity regardless of the CNT weight ratio. Therefore, we may pay attention to the radius-length effects 


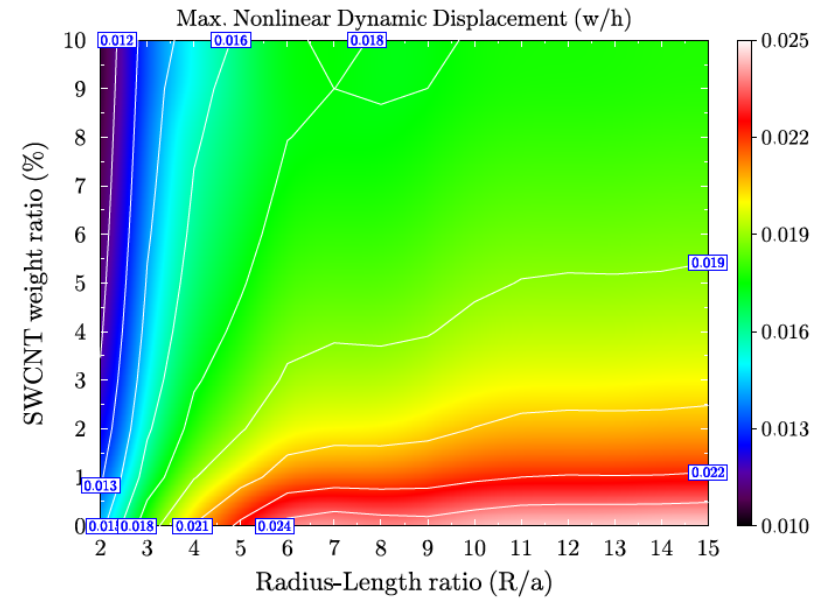

(a) $\mathrm{c} / \mathrm{a}=0.1$

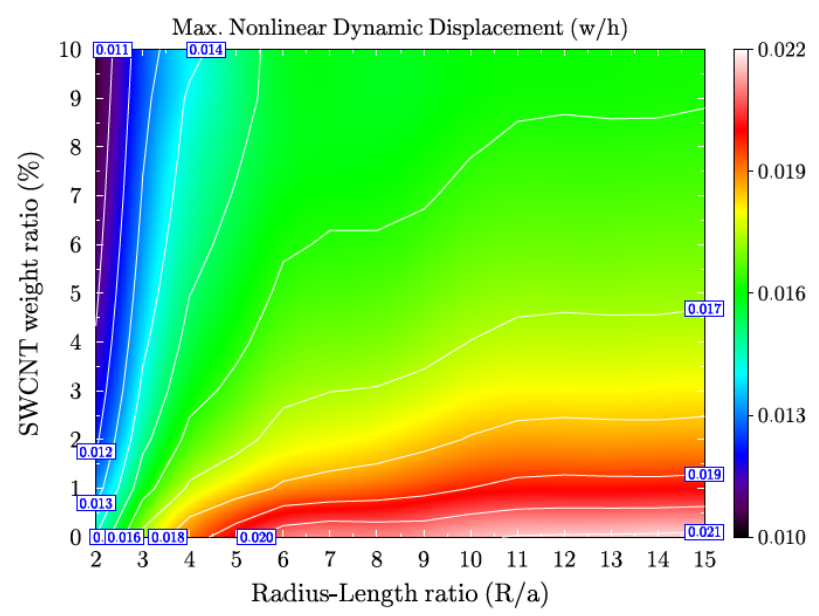

(c) $\mathrm{c} / \mathrm{a}=0.4$

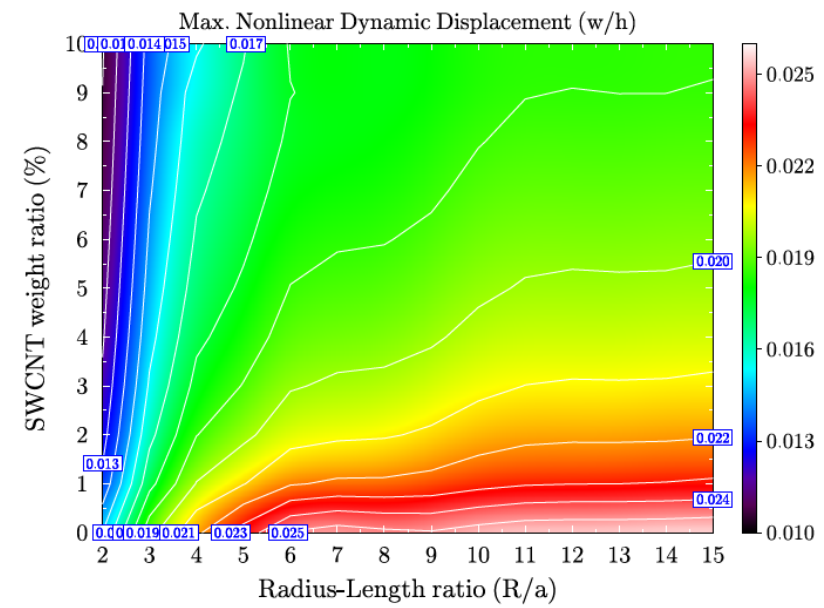

(b) $\mathrm{c} / \mathrm{a}=0.2$

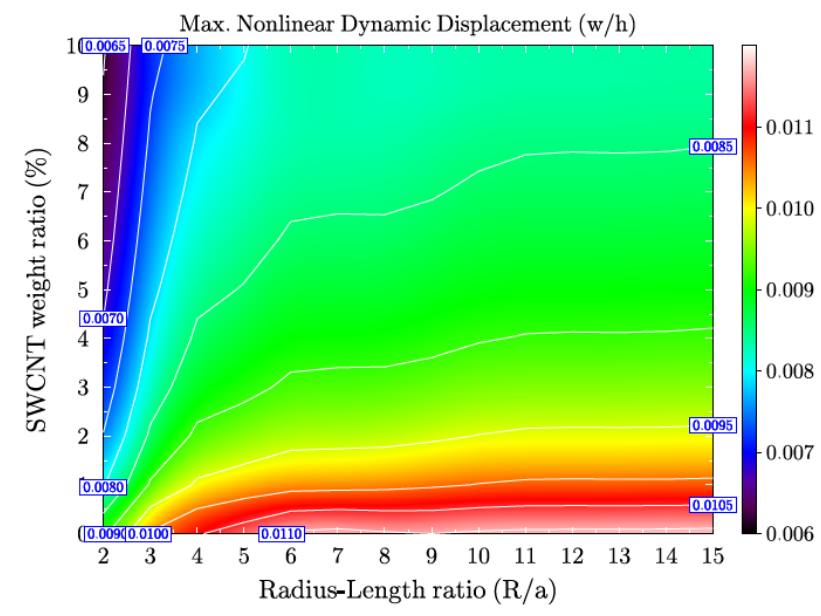

(d) $\mathrm{c} / \mathrm{a}=0.6$

Figure 9: Maximum nonlinear dynamic displacements of [45/-45/45/-45] CNTFPC spherical shells for increased cutout sizes and SWCNT weight ratios, and radius-length ratios

of spherical CNTFPC shells because the various geometrical nonlinear dynamic behaviors made by the cutout area could be significant to the curvature of spherical shells containing a cutout.

\section{Summary and conclusion}

In this study, we performed a nonlinear transient analysis of laminated CNTFPC spherical shells containing a central cutout according to the FSDT model. Multi-scale formulations of SWCNT or MWCNT reinforced three-phase composites were investigated by comparison with the conventional and modified Halpin-Tsai models. From the numeri- cal results, the following significant observations and conclusions were drawn:

1. For volume fractions of more than 0.6, the differences in induced natural frequencies between the conventional and the modified Halpin-Tasi models were significant for increased SWCNT or MWCNT weight ratios. Therefore, the modified Halpin-Tsai model is recommended for greater accuracy, especially for the high volume fractions.

2. The maximum nonlinear dynamic displacement occurred at lower disturbing frequencies as the radiusthickness ratios decreased. The membrane effects in the spherical shell led to increased stiffness and resulted in higher excitation frequency. For shells with a cutout, the excitation frequencies were lower than those for shells without a cutout, because the area of 
the shells decreased. Small SWCNT weight ratios of $1-2 \%$ play a significant role in the downward shift of the nonlinear dynamic displacement.

3. Maximum displacements increased as the cutout areas increased because of the effects of the reduced stiffness. On the other hand, the SWCNT reinforcement produced higher nonlinear frequencies for increased curvature effects. Only $2 \%$ of the CNTreinforcement for shells with $40 \%$ cutout areas affected the nonlinear dynamic rigidity similarly to that for panels with $10 \%$ cutout areas. The SWCNT reinforcement enables the nonlinear dynamic rigidity of spherical CNTFPC shells with a cutout to be enhanced.

4. From the parametric results, spherical shells with $R / a>5.0$ can be considered as a similar nonlinear dynamic flexural rigidity regardless of the CNT weight ratios. However, we cannot neglect the effect of the thickness-length ratio in analyzing the spherical CNTFPC shells because the various effects of the cutout area could be significant to the thickness or length of spherical shells containing a cutout.

Acknowledgement: This work was supported by the National Research Foundation of Korea (NRF) grant funded by the Korea government (MSIP - Korea) (No. 2018R1D1A1B07050080).

\section{References}

[1] Rafi S.S., Anusha P., Sri M.N.S., Estmation of Young's Modulus for Single Walled Carbon Nanotube With Finite Element Method, Int. J. Eng. Res. Technol., 2014, 3(5), 54-62.

[2] Yeh M.K., Tai N.H., Liu J.H., Mechanical behavior of phenolicbased composites reinforced with multi-walled carbon nanotubes, Carbon, 2006, 44, 1-9.

[3] Han Y., Elliott J., Molecular dynamics simulations of the elastic properties of polymer/carbon nanotube composites, Comput. Mater. Sci., 2007, 39, 315-323.

[4] Omidi M., Rokni H., Milani A.S., Seethaler R. J., Arasteh R., Prediction of the mechanical characteristics of multi-walled carbon nanotube/epoxy composites using a new form of the rule of mixtures, Carbon, 2010, 48, 3218-3228.

[5] Thostenson E.T., Li C., Chou T.-W., Nanocomposites in context, Compos. Sci. Technol., 2005, 65, 491516.

[6] Wattanasakulpong N., Ungbhakorn V., Analytical solutions for bending, buckling and vibration responses of carbon nanotubereinforced composite beams resting on elastic foundation, Comput. Mater. Sci., 2013, 71, 201-208.

[7] Zhu P., Lei Z.X., Liew K.M., Static and free vibration analyses of carbon nanotube-reinforced composite plates using finite element method with first order shear deformation plate theory, Compos. Struct., 2012, 94, 1450-1460.
[8] Zhang L.W., Song Z.G., Liew K.M., State-space Levy method for vibration analysis of FG-CNT composite plates subjected to inplane loads based on higher-order shear deformation theory, Compos. Struct., 2015, 134, 9891003.

[9] Rafiee M., Liu X.F., He X.Q., Kitipornchai S., Geometrically nonlinear free vibration of shear deformable piezoelectric carbon nanotube/fiber/polymer multiscale laminated composite plates, J. Sound Vibr., 2014, 333, 3236-3251.

[10] Garca-Macas E., Rodriguez-Tembleque L., Castro-Triguero R., Sez A., Buckling analysis of functionally graded carbon nanotubereinforced curved panels under axial compression and shear, Compos. Part B: Eng., 2017, 108, 243-256.

[11] Mirzaei M., Kiani Y., Free vibration of functionally graded carbon nanotube reinforced composite cylindrical panels. Compos, Struct., 2016, 142, 45-56.

[12] Zhang L.W., Lei Z.X., Liew K.M., Yu J.L., Static and dynamic of carbon nanotube reinforced functionally graded cylindrical panels. Compos. Struct., 2014, 111, 205-212.

[13] Lei Z.X., Zhang L.W., Liew K.M., Yu J.L., Dynamic stability analysis of carbon nanotube-reinforced functionally graded cylindrical panels using the element-free kp-Ritz method, Compos. Struct., 2014, 113, 328-338.

[14] Asadi H., Wang Q., Dynamic stability analysis of a pressurized FG-CNTRC cylindrical shell interacting with supersonic airflow, Compos. Part B: Eng., 2017, 118, 15-25.

[15] Bidgoli M.R., Karimi M.S., Arani A.G., Nonlinear vibration and instability analysis of functionally graded CNT-reinforced cylindrical shells conveying viscous fluid resting on orthotropic Pasternak medium, Mech. Adv. Mater. Struct., 2016, 23, 819-831.

[16] Bhardwaj G., Upadhyay A.K., Pandey R., Shukla K.K., Non-linear flexural and dynamic response of CNT reinforced laminated composite plates, J. Compos. Part B, 2013, 45, 89-100.

[17] Nanda N., Bandyopadhyay J.N., Geometrically nonlinear transient analysis of laminated composite shells using the finite element method, J. Sound Vibr., 2009, 325, 174-185.

[18] Reddy J.N., Chandrashekhara K., Geometrically nonlinear transient analysis of laminated, doubly curved shells, Int. J. Nonlin. Mech., 1985, 20(2), 77-90.

[19] Han S.C., Tabiei A., Park W.T., Geometrically nonlinear analysis of laminated composite thin shells using a modified first-order shear deformable element-based Lagrangian shell element, Compos. Struct., 2008, 82, 465-474.

[20] Gojny F.H., Wichmann M.H.G., Kpke U., Fiedler B., Schulte K., Carbon nanotube-reinforced epoxy-composites enhanced stiffness and fracture toughness at low nanotube content, Compos. Sci. Technol., 2004, 64, 2363-2371.

[21] Hashin Z., Theory of fiber reinforced materials, NASA Tech. Rep., 1970, contract no: NAS1-8818.

[22] Sanders J.L., Nonlinear theories for thin shells, Quart. Appl. Math., 1963, 21, 21-36.

[23] Park T., Lee S.Y., Parametric instability of delaminated composite spherical shells subjected to in-plane pulsating forces, Compos. Struct., 2009, 91, 196-204.

[24] Kurtaran H., Geometrically nonlinear transient analysis of moderately thick laminated composite shallow shells with generalized differential quadrature method, Compos. Struct., 2015, 125, 605614.

[25] Kim M., Park Y.B., Okoli O.I., Zhang C., Processing, characterization, and modeling of carbon nanotube-reinforced multiscale composites, Compos. Sci. Technol., 2009, 69, 335-342. 